\title{
Cognition of DTN Wireless Relay Multicast Routing through Link Reliability Relation
}

\author{
Dening Kong \\ Wuhan Digital Engineering Institute, Wuhan , 430074 China \\ Email: 1569244672@qq.com
}

Keywords: DTN wireless network; multicast; relay routing; link reliability

\begin{abstract}
The main objective of DTN routing is to transfer the message to the largest extent. In another words, the delivery ratio is the most important evaluation index of DTN routing. The priority of data package in DTN network is the degree of importance of the data package and data packages with different priorities shall have different delivery ratios to differentiate their degree of importance. RMRP algorithm in the Thesis achieves the service differentiation by priority on the basis of ensuring that data with various priorities can be delivered with their respective probability in accordance with the forwarding probability of each node. The experiment indicates that the network performance sacrificed by the data with high priority can be made up by data with low priority. If the difference between the volume of data with high and low priority is not significant, the performance can be maintained stable while the whole DTN network achieves service differentiation.
\end{abstract}

\section{Introduction}

Internet architecture and many protocols cannot be better applicable to network with high-delay route and frequent disruption. For example, land mobile network, military wireless self-organizing network, planetary network and sensor and this kind of network is called limited network [1]. However, the fact that the communication source and object in limited network have no complete connection route does not mean that it can not realize communication. With the movement of nodes, two nodes can enter the intercommunication range and exchange data [2]. In the existing DTN routing algorithm, there are several kinds based on service differentiation by priority. In Literature [3], the maximum copy number of message is expressed as the initial priority function of the message. Based on the difference of priority, the data with high priority will forward more backup copies with fixed quantity to the node meeting during the forwarding to improve the delivery ratio. However, the algorithm does not differentiate forwarding strategy in accordance with the difference of forwarding characteristics of each node. In Literature [4-6], the network congestion caused by the exhaustion of cache resources of nodes due to the custody of transport protocol in DTN is reduced through the method of reserving cache resources for subsequent business with high priority so as to improve the quality of service. However, the algorithm will cause serious delay of data with low priority and packet loss probability when the node cache is low or the coming of data with high priority is frequent, seriously influencing the transmission of data with low priority.

\section{Algorithm design}

Calculation of forwarding probability

In the architecture of DTN, the bit layer of the process control mark in BUNDLE layer defines the priority of data with the two bytes 7 and 8:

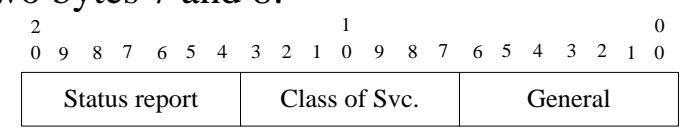

Fig.1 Bit Layer of the Process Control Mark in BUNDLE Layer

It is divided into three grades: $00=$ low priority, $01=$ medium priority and $10=$ high priority. All routing algorithms of DTN differentiating priority define three different kinds of priority based on this and the work in the Thesis is carried out based on the three kinds of priority defined. After the 
identification of priority of data package, corresponding routing strategy shall be taken to realize the function of service differentiation.

The RMRP algorithm presented in the Thesis adopts the calculation and updating method of the traditional algorithm at the forwarding probability calculation stage and it calculates the mutual forwarding probability when different nodes meet. The following three situations are mainly included:

1. When two node meet and they are in the communication range of each other, the forwarding node will update its own forwarding probability and the formula for the updating of forwarding probability is as follows

$$
P_{(\mathrm{a}, \mathrm{b})}=P_{(\mathrm{a}, \mathrm{b}) \text { old }}+\left(1-P_{(\mathrm{a}, \mathrm{b}) \text { old }}\right) \times P_{\text {init }}
$$

$P_{\text {init }}$ is the initial constant set. It can be seen from Formula (1) that if the forwarding node meets other nodes frequently, its forwarding probability is relatively high.

2. After a period of time, if the two nodes do not meet or they are not in the communication range of each other, the forwarding probability of the forwarding node will reduce correspondingly and the formula for the updating of probability is as follows

$$
\begin{aligned}
& P_{(\mathrm{a}, \mathrm{b})}=P_{(\mathrm{a}, \mathrm{b}) \text { old }} \times \gamma^{k} \\
& \gamma \in[0,1) \text { is attenuation }
\end{aligned}
$$

time. Thus it can be seen that when the forwarding node does not meet other nodes within certain period of time, its forwarding probability will reduce.

3. If the two nodes a and b meet frequently and two nodes b and c meet frequently, then $\mathrm{c}$ is the good relay node of a and the formula for the updating of probability between a and $\mathrm{c}$ is as follows

$$
P_{(\mathrm{a}, \mathrm{c})}=P_{(\mathrm{a}, \mathrm{c}) \text { old }}+\left(1-P_{(\mathrm{a}, \mathrm{c}) \text { old }}\right) \times P_{(\mathrm{a}, \mathrm{b})} \times P_{(\mathrm{b}, \mathrm{c})}
$$

That is to say, the forwarding probability between nodes is of transfer attribute.

Message forwarding process

To differentiate services with different priorities, the concept of reference probability is presented in the Thesis. During the forwarding of data package with high priority, the forwarding probability shall be increased to improve the reachability. If node a sends message to node $b$, compare the forwarding probability of $\mathrm{b}{ }^{P_{(b, d)}}$ with the reference probability of node a $P_{r}$, if $P_{(b, d)}>P_{r}$, forward the message; otherwise, do not forward the message. The reference probability is defined as

$$
P_{r}=C \cdot P_{(a, d)} \quad(4)
$$

When the priority is high, $\mathrm{C}<1$; when the priority is medium, $\mathrm{C}=1$; when the priority is low, $\mathrm{C}>1$. It can be seen from Formula (4) that when the priority is different, the reference probability is different correspondingly. When the data package is of high priority, the reference probability is small. In another word, the reference threshold value of the forwarding probability of nodes meeting is low and more data packages will meet the requirements for forwarding and achieve the forwarding so as to improve the delivery ratio. When the data package is of medium priority, the reference probability is equal to the forwarding probability of node a. When the data package is of low priority, the reference threshold value is high and less data package copies are transmitted in the network and the delivery ratio is reduced.

The reference probability in network forwarding is related to the bandwidth and size of message of link. The priority of data package is defined as 3, 2, and 1 from high to low which are expressed by letter ${ }^{x_{h}},{ }^{x_{m}}$ and ${ }^{x_{l}}$, where, ${ }^{x}$ is the priority of any data package. Then, the value of $\mathrm{C}$ is defined as

$$
\mathrm{C}=1+0.1\left(x_{m}-x\right) \frac{x_{m}^{2}}{x+1}\left(\frac{B}{L}\right)^{x-x_{m}}
$$

$\mathrm{B}$ is link bandwidth and $\mathrm{L}$ is the size of message. When ${ }^{x}=x_{h}$, i.e.: high priority, $\mathrm{C}<1$. When $x=x_{m}$, i.e.: medium priority, $\mathrm{C}=1$. When ${ }^{x}=x_{l}$, i.e.: low priority, $\mathrm{C}>1$. It can be analyzed from Formula (5) that, if the bandwidth is high or the message is small during the data package transmission, the network redundancy is relatively large and the link is relatively leisure. Then, the 
requirement for reference threshold value can be reduced and the value of $\mathrm{C}$ can also be reduced to transmit more data package copies so as to improve the delivery ratio of data and use ratio of network, vice versa.

\section{Experiment and analysis}

\section{Experimental environment}

Indexes for the evaluation of DTN routing algorithm are generally delivery ratio and load ratio and the network load and delivery utility index presented in Literature [13] also evaluate the network performance from different aspects. In the Thesis, the above-mentioned four indexes are used to analyze the performance of algorithm. ONE simulation platform is the discrete event simulator based on Java and it is designed for DTN protocol frame and it can realize the simulation of mobile model, routing algorithm and application protocol. In the Thesis, ONE simulation platform is used for the experiment and RMRP algorithm is applied to the platform to achieve differentiation of services with different priorities and it is compared with the traditional algorithm. The default scenario in ONE is adopted as the analogue simulation scenario and the size of the simulated area is $4500 \mathrm{~m} * 3400 \mathrm{~m}$ which consists of 126 nodes. Nodes are divided into three groups and they are set up in accordance with the difference of characteristics of three groups of nodes. The first group consists of nodes of pedestrian walking in which the speed is $0.5-1.5 \mathrm{~m} / \mathrm{s}$, node cache is $50 \mathrm{M}$ and the number of nodes is 80 . The second group consists of nodes of car driving in which the speed is $2.7-13.9 \mathrm{~m} / \mathrm{s}$, the node cache is $50 \mathrm{M}$ and the number of nodes is 40 . The third group consists of nodes of tramcar traveling in which the speed is $7-10 \mathrm{~m} / \mathrm{s}$, the node cache is $50 \mathrm{M}$ and the number of nodes is 6.

Simulation result

Use simulation platform to simulate the above-mentioned scenarios and conduct comparative analysis between the RMRP algorithm and traditional method and compare data packages with different priorities in accordance with different indexes.

It can be seen from Fig. 2 and Fig.3 that: in RMRP algorithm, under different TTL, the delivery ratio of three kinds of data package shows layered distribution in accordance with the difference of priority-low, medium and high. However, the traditional algorithm is not sensitive to the priority of data package and it does not differentiate services for data package based on different priorities and the delivery ratio of data packages with different priorities is basically the same. In addition, when TTL decreases, part of data packages in the cache is unable to realize the delivery of data in the high-delay DTN network due to the insufficiency of TTL. Therefore, during the decrease process of TTL, the delivery ratio is on a declining curve as a whole.

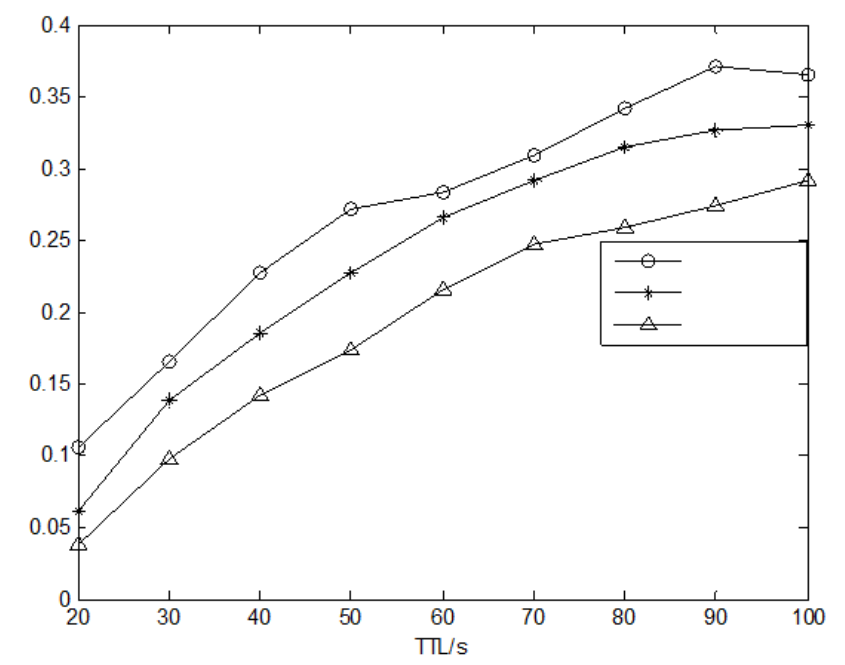

Fig.2 Comparison of Delivery Ratio of RMRP Algorithm 


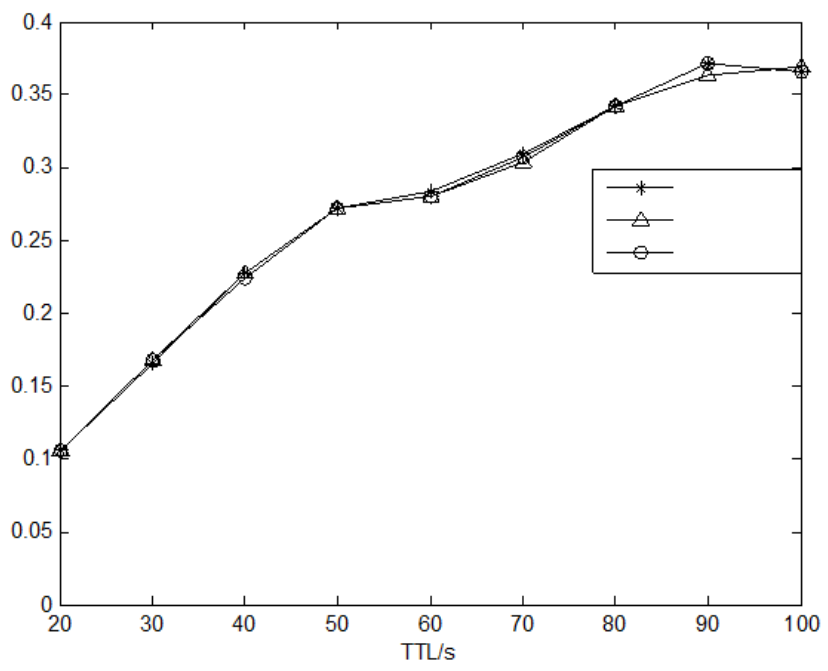

Fig.3 Comparison of Delivery Ratio of Traditional Algorithm

The load ratio describes the repeatability of data delivered to the destination node. In the network transmission quality evaluation, there is a negative correlation between the transmission efficiency and load ratio. It can be seen from Fig.4 that: in RMRP algorithm, the data package with low priority has lower load ratio while the transmission efficiency is higher. The data package with high priority has higher load ratio while the transmission efficiency is lower.

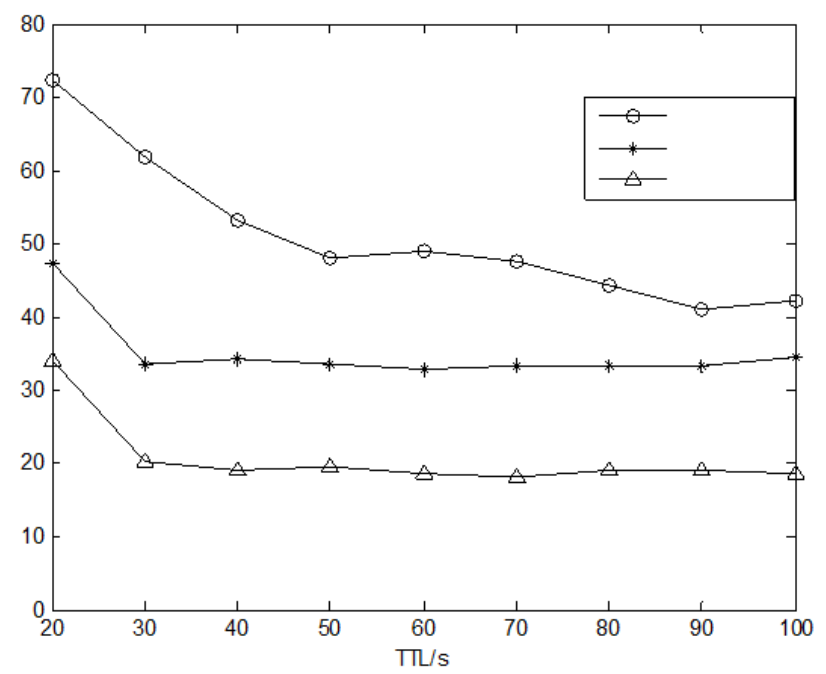

Fig.4 Comparison of Load Ratio of RMRP Algorithm

It can be known through the analysis of the above-mentioned experiment that: in RMRP algorithm, data with different priorities has different delivery ratio in network transmission and the higher the priority is, the larger the forwarding probability, realizing the function of differentiating service by priorities. Through the analysis for the performance, the delivery ratio of data package with high priority is improved but it will cause heavy network load, reduction of transmission utility and increase of load ratio (repetitive rate of data reached). The delivery ratio of data package with low priority is decreased but it reduces the burden of network and the transmission utility is increased greatly and the repetitive rate of data reached is reduced, balancing the overall performance of the whole network.

Fig. 5 shows the number of data copies generated during the transmission of data package with different priorities in the network. As different priorities make no difference to the number of copies forwarded in the traditional algorithm, the number of data copies of data with medium priority in the RMRP algorithm and traditional algorithm is the same. It can be seen through the comparison based on the figure that the data with high priority in RMRP algorithm generates more copies and bring about high load to the network while the data with low priority in RMRP algorithm reduces 
the number of copies in the network and reduces the network load.

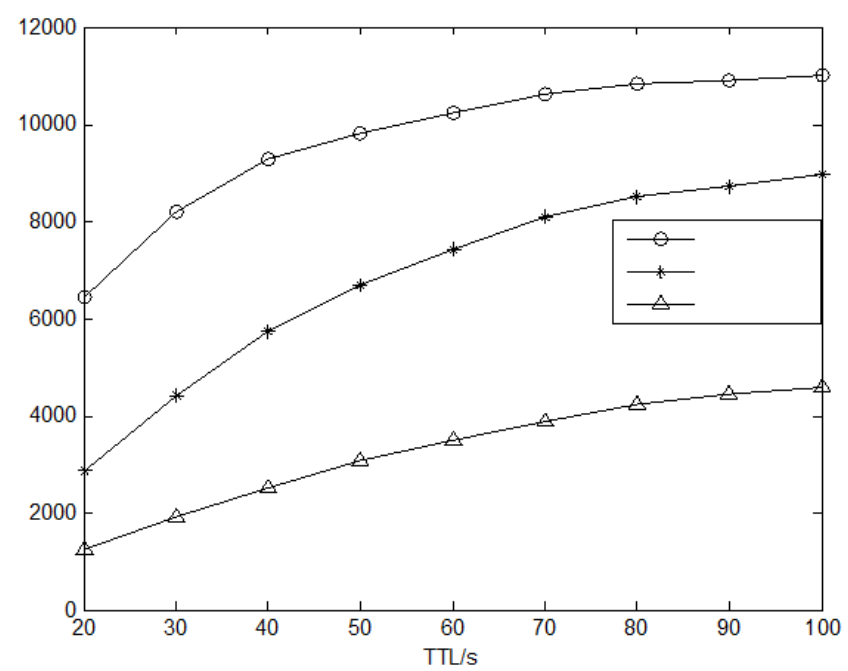

Fig.5 Comparison of Network Load of RMRP Algorithm

\section{Conclusion}

For the issue that data package with different priorities in DTN network requires service differentiation, the probability routing algorithm RMRP which can differentiate services is presented. The Algorithm proposes the concept of reference probability and the nodes meeting with each other define different reference probabilities based on different priorities of data package. If the reference probability of nodes meeting is larger than the forwarding probability of the sending node, the data package will be forwarded; otherwise, it will not be forwarded. The simulation indicates that RMRP algorithm makes the delivery ratio of data package with different priorities show layered distribution and the delivery ratio of data package with high, medium and low priority arranges from high to low. The Algorithm enables DTN network to better achieve the function of differentiating services in accordance with the difference of priority of data package without changing the communication performance of the original network.

\section{Reference}

[1] Yishuang Geng, Jin Chen, Ruijun Fu, Guanqun Bao, Kaveh Pahlavan, Enlighten wearable physiological monitoring systems: On-body rf characteristics based human motion classification using a support vector machine, IEEE transactions on mobile computing, 1(1), 1-15, Apr. 2015

[2] Jiang, D., Xu, Z., \& Lv, Z. (2015). A multicast delivery approach with minimum energy consumption for wireless multi-hop networks. Telecommunication systems, 1-12.

[3] Lv, Z., Chirivella, J., \& Gagliardo, P. (2016). Bigdata Oriented Multimedia Mobile Health Applications. Journal of medical systems, 40(5), 1-10.

[4] Jinyu Hu, Zhiwei Gao and Weisen Pan. Multiangle Social Network Recommendation Algorithms and Similarity Network Evaluation[J]. Journal of Applied Mathematics, 2013 (2013).

[5] Lv, Z., Halawani, A., Feng, S., Li, H., \& Réhman, S. U. (2014). Multimodal hand and foot gesture interaction for handheld devices. ACM Transactions on Multimedia Computing, Communications, and Applications (TOMM), 11(1s), 10.

[6] Yang, J., Wang, H., Lv, Z., Wei, W., Song, H., Erol-Kantarci, M., ... \& He, S. (2016). Multimedia recommendation and transmission system based on cloud platform. Future Generation Computer Systems. 